\title{
Green Function Estimates and Harnack Inequality for Subordinate Brownian Motions
}

\author{
MURALI RAO ${ }^{1}$, RENMING SONG ${ }^{2}$ and ZORAN VONDRAČKK ${ }^{3}$ \\ ${ }^{1}$ Department of Mathematics, University of Florida, Gainesville, FL 32611, U.S.A. \\ (e-mail:rao@math.ufl.edu) \\ ${ }^{2}$ Department of Mathematics, University of Illinois, Urbana, IL 61801, U.S.A. \\ (e-mail:rsong@math.uiuc.edu) \\ ${ }^{3}$ Department of Mathematics, University of Zagreb, Zagreb, Croatia (e-mail: vondra@math.hr)
}

(Received: 1 December 2004; accepted: 13 October 2005)

\begin{abstract}
Let $X$ be a Lévy process in $\mathbb{R}^{d}, d \geqslant 3$, obtained by subordinating Brownian motion with a subordinator with a positive drift. Such a process has the same law as the sum of an independent Brownian motion and a Lévy process with no continuous component. We study the asymptotic behavior of the Green function of $X$ near zero. Under the assumption that the Laplace exponent of the subordinator is a complete Bernstein function we also describe the asymptotic behavior of the Green function at infinity. With an additional assumption on the Lévy measure of the subordinator we prove that the Harnack inequality is valid for the nonnegative harmonic functions of $X$.
\end{abstract}

Mathematics Subject Classifications (2000): Primary 60J45, 60J75, Secondary 60J25.

Key words: Subordinate Brownian motion, Green function, Harnack inequality, subordinator, complete Bernstein function, capacity.

\section{Introduction}

Let $X=\left(X_{t}, \mathbb{P}_{x}, x \in \mathbb{R}^{d}\right)$ be a conservative strong Markov process on $\mathbb{R}^{d}$. A nonnegative Borel function $h$ on $\mathbb{R}^{d}$ is said to be harmonic with respect to $X$ in a domain (i.e., a connected open set) $D \subset \mathbb{R}^{d}$ if it is not identically infinite in $D$ and if for any bounded open subset $B \subset \bar{B} \subset D$,

$$
h(x)=\mathbb{E}_{x}\left[h\left(X\left(\tau_{B}\right)\right) 1_{\tau_{B}<\infty}\right], \quad \forall x \in B,
$$

where $\tau_{B}=\inf \left\{t>0: X_{t} \notin B\right\}$ is the first exit time of $B$.

We say that the Harnack inequality holds for $X$ if for any domain $D \subset \mathbb{R}^{d}$ and any compact subset $K$ of $D$, there is a constant $C>0$ depending only on $D$ and $K$ such that for any nonnegative function $h$ harmonic with respect to $X$ in $D$,

$$
\sup _{x \in K} h(x) \leqslant C \inf _{x \in K} h(x) .
$$


It is well known that the Harnack inequality holds true for many Markov processes with continuous paths. A probabilistic proof for diffusions generated by elliptic operators in non-divergence form was given by Krylov and Safonov [15]. The proof is based on the fact that the diffusion hits sets with positive Lebesgue measure with positive probability. Until very recently almost all results concerning the Harnack inequality were restricted to Markov processes with continuous paths, i.e., to harmonic functions corresponding to local operators. The only exception was the rotationally invariant $\alpha$-stable process, $\alpha \in(0,2)$, in which case the Harnack inequality follows directly from the explicit form of the exit distribution from a ball (i.e., the corresponding Poisson kernel) and was proved in [17].

A serious study of the Harnack inequality for discontinuous Markov processes on $\mathbb{R}^{d}$ began with the paper [3] by Bass and Levin. They proved the Harnack inequality for the Markov process on $\mathbb{R}^{d}$ associated with the generator

$$
L f(x)=\int_{R^{d} \backslash\{0\}}[f(x+y)-f(x)] \frac{k(x, y)}{|y|^{d+\alpha}} \mathrm{d} y
$$

where $k(x,-y)=k(x, y)$ and $k$ is a positive function bounded between two positive numbers. Vondraček [27] adapted the arguments of Bass and Levin [3] and proved that, when $X$ is a (not necessarily rotationally invariant) strictly $\alpha$ stable process, $\alpha \in(0,2)$, with a Lévy measure comparable to the Lévy measure of the rotationally invariant $\alpha$-stable process, the Harnack inequality holds. In [8], the Harnack inequality was proved by using a different method for symmetric $\alpha$-stable processes under the assumptions that $\alpha \in(0,1)$ and its Lévy measure is comparable to the Lévy measure of the rotationally invariant $\alpha$-stable process. In their work, Bass and Levin [4] established upper and lower bounds on the transition densities of symmetric Markov chains on the integer lattice in $d$ dimensions, where the conductance between $x$ and $y$ is comparable to $|x-y|^{d+\alpha}$, $\alpha \in(0,2)$. One of the key steps in proving the upper and lower bounds in [4] is the parabolic Harnack inequality. Chen and Kumagai [9] showed that the parabolic Harnack inequality holds for symmetric stable-like processes in $d$ sets and established upper and lower bounds on the transition densities of these processes. All the processes mentioned above satisfy a certain scaling property which was used crucially in the proofs of the Harnack inequalities. In their paper, Song and Vondraček [24] extracted the essential ingredients of the Bass-Levin method by isolating three conditions that suffice to prove the Harnack inequality and showed that various classes of Markov processes, not necessarily having any scaling properties, satisfy the Harnack inequality. In a recent paper [2], Bass and Kassmann proved the Harnack inequality for a class of processes corresponding to non-local operators of variable order. Their method is also based on [3], but the arguments are more delicate, yielding a weaker form of the Harnack inequality for balls in the sense that the constant may depend on the radius of the ball. 
A common feature of all the papers mentioned above is that they all deal with purely discontinuous processes. To the best of our knowledge, except for the very recent paper [25], the Harnack inequality has not been established for discontinuous Markov processes with a nondegenerate diffusion part. In this paper we try to fill this gap by considering subordinate Brownian motion using a subordinator with a positive drift term.

Subordination of Markov processes has a long history going back to Bochner. The renewed interest in subordination in recent years has come from different directions. In [12] (see also the references therein) it has been argued that asset price processes should be modelled as subordinate Brownian motion instead of Brownian motion itself. With this application comes the need to study properties of subordinate Brownian motions. Connections of the subordinate Brownian motion (using stable subordinators) with pseudo differential operators were explored by Jacob and Schilling [14]. Some potential-theoretic aspects of the subordinate killed Brownian motion (using stable subordinators) were studied in $[22,23]$. Connections of subordinate processes with jump processes on fractal were studied by Kumagai in [16] and Stós in [26].

A subordinator $T=\left(T_{t}: t \geqslant 0\right)$ is a nondecreasing Lévy process with the state space $[0, \infty)$. The law of $T$ is characterized by its Laplace exponent $\phi$ through the relation $\mathbb{E}\left[\exp \left\{-\lambda T_{t}\right\}\right]=\exp \{-t \phi(\lambda)\}$. In this paper we will consider Lévy processes $X=\left(X_{t}: t \geqslant 0\right)$ in $\mathbb{R}^{d}, d \geqslant 3$, that can be obtained by subordinating a $d$-dimensional Brownian motion $Y=\left(Y_{t}: t \geqslant 0\right)$ by the subordinator $T$. To be more precise, we define $X_{t}:=Y\left(T_{t}\right)$. Our main interest will be in subordinators with a non-zero drift of the type $T_{t}:=b t+\tilde{T}_{t}$, where $b>0$ and $\tilde{T}$ is a pure jump subordinator. In this case the subordinate process $X$ has the same law as the independent sum of a Brownian motion and a Brownian motion subordinate by $\tilde{T}$. A prominent example of such a process is a sum of independent Brownian motion and a rotationally invariant $\alpha$-stable process.

It is well known that for the pure jump subordinator $\tilde{T}_{t}$ we have $\lim _{t \rightarrow 0} \tilde{T}_{t} / t=$ 0 a.s. Therefore, the small time behavior of the subordinator $T_{t}=t+\tilde{T}_{t}$ is determined by the drift. As a consequence, the small time behavior of the subordinate process $X_{t}=Y\left(T_{t}\right)$ should be similar to the small time behavior of Brownian motion $Y$. We make this statement precise by showing that the Green function $G(x, y)$ of $X$ is asymptotically equal to the Green function of the Brownian motion as $|x-y| \rightarrow 0$. We obtain this result from the asymptotic behavior of the Laplace exponent of $T$ at infinity. Another advantage of subordination is that the Lévy measure of $X$ can be described explicitly in terms of the Lévy measure of $T$, leading to properties of the former needed for the proof of the Harnack inequality.

The paper is organized as follows. In Section 2 we recall some properties of subordinators, in particularly the connection with the Bernstein functions and complete Bernstein functions. In Section 3 we introduce the subordinate process $X$ and study the asymptotic behavior of its Green function at 0 and at infinity. 
The subordinators that we use include subordinators with drift, but are more general. As a consequence of the behavior of the Green function at 0 , we obtain the asymptotics of the exit times from small balls as well as capacity of small balls. In Section 4 we prove the Harnack inequality for the nonnegative harmonic functions of $X$ by use of a modified approach from [3]. In the last section we briefly indicate how some of our results extend to the case in which Brownian motion is replaced by a uniformly elliptic diffusion or a fractal diffusion.

In the paper we use following notation: If $f$ and $g$ are two functions, then $f \sim g$ as $x \rightarrow 0$ (respectively, $x \rightarrow \infty$ ), if $(f / g)(x)$ converges to 1 as $x$ converges to 0 (respectively, $x$ converges to $\infty$ ), and we write $f \asymp g$ if the quotient $f / g$ stays bounded between two positive constants.

\section{Subordinators and Complete Bernstein Functions}

Let $T=\left(T_{t}: t \geqslant 0\right)$ be a subordinator, i.e., a Lévy process taking values in $[0, \infty)$ and having almost surely non-decreasing paths. The Laplace transform of the law of $T$ is given by the formula

$$
\mathbb{E}\left[\exp \left(-\lambda T_{t}\right)\right]=\exp (-t \phi(\lambda)), \quad \lambda>0 .
$$

The function $\phi:(0, \infty) \rightarrow \mathbb{R}$ is called the Laplace exponent, and its form is given by

$$
\phi(\lambda)=b \lambda+\int_{0}^{\infty}\left(1-\mathrm{e}^{-\lambda t}\right) \nu(\mathrm{d} t) .
$$

Here $b \geqslant 0$, and $\nu$ is a $\sigma$-finite measure on $(0, \infty)$ satisfying

$$
\int_{0}^{\infty}(t \wedge 1) \nu(\mathrm{d} t)<\infty
$$

The constant $b$ is called the drift and $\nu$ the Lévy measure of the subordinator $T$.

Recall that a $C^{\infty}$ function $\phi:(0, \infty) \rightarrow[0, \infty)$ is called a Bernstein function if $(-1)^{n} D^{n} \phi \leqslant 0$ for every $n \in \mathbb{N}$. It is well known that a function $\phi:(0, \infty) \rightarrow$ $\mathbb{R}$ is a Bernstein function if and only if it has the representation given below

$$
\phi(\lambda)=a+b \lambda+\int_{0}^{\infty}\left(1-\mathrm{e}^{-\lambda t}\right) \nu(\mathrm{d} t)
$$

where $a, b \geqslant 0$, and $\nu$ is a $\sigma$-finite measure on $(0, \infty)$ satisfying (2.3).

The potential measure of the subordinator $T$ is defined by

$$
U(A)=\mathbb{E} \int_{0}^{\infty} 1_{\left(T_{t} \in A\right)} \mathrm{d} t,
$$


and its Laplace transform is given by

$$
\mathcal{L} U(\lambda)=\mathbb{E} \int_{0}^{\infty} \exp \left(-\lambda T_{t}\right) \mathrm{d} t=\frac{1}{\phi(\lambda)} .
$$

We will be interested in subordinators with absolutely continuous potential measures. Suppose that the drift coefficient $b$ is positive. Then the potential measure is absolutely continuous with a density $u:(0, \infty) \rightarrow \mathbb{R}$ that is continuous and positive, and $u(0+)=1 / b$ (e.g., [6], p. 79).

LEMMA 2.1. Let $T=\left(T_{t}: t \geqslant 0\right)$ be a subordinator with the Laplace exponent given by (2.2). Suppose that $b>0$, and let $u$ be the potential density of $T$. Then $u(0+) \geqslant u(x)$ for every $x>0$.

Proof. Recall that $x \rightarrow U([0, x])$ is subadditive ([6], p. 74). Thus, for all $x>0, r>0$,

$$
U([x, x+r])=U([0, x+r])-U([0, x]) \leqslant U([0, r]),
$$

which can be rewritten as

$$
\int_{x}^{x+r} u(y) \mathrm{d} y \leqslant \int_{0}^{r} u(y) \mathrm{d} y .
$$

Dividing by $r$, letting $r \rightarrow 0$, and by using continuity of $u$, it follows that $u(x) \leqslant$ $u(0+)$.

A function $\phi:(0, \infty) \rightarrow \mathbb{R}$ is called a complete Bernstein function if there exists a Bernstein function $\psi$ such that

$$
\phi(\lambda)=\lambda^{2} \mathcal{L} \psi(\lambda), \quad \lambda>0,
$$

where $\mathcal{L}$ stands for the Laplace transform. The following result is due to Schilling [20], and its proof can also be found in [13] (pp. 192-193).

LEMMA 2.2. Let $\phi$ be a complete Bernstein function with $\phi(\lambda)=\lambda^{2} \mathcal{L} \psi(\lambda)$. If the Bernstein function $\psi$ has the representation

$$
\psi(\lambda)=a+b \lambda+\int_{0}^{\infty}\left(1-\mathrm{e}^{-\lambda t}\right) \mu(\mathrm{d} t),
$$

then $\phi$ has the representation

$$
\phi(\lambda)=b+a \lambda+\int_{0}^{\infty}\left(1-\mathrm{e}^{-\lambda t}\right) \nu(\mathrm{d} t)
$$


with an absolutely continuous Lévy measure $\nu$. In particular, $\phi$ is a Bernstein function.

The family of all complete Bernstein functions is a convex cone containing positive constants and it is closed under compositions. Most of the familiar Bernstein functions are complete Bernstein functions. The following are some examples of complete Bernstein functions ([13]):

$$
\begin{aligned}
& \lambda^{\alpha}, \alpha \in(0,1], \\
& (\lambda+1)^{\alpha}-1, \\
& \log (1+\lambda), \\
& \frac{\lambda}{\lambda+1}, \\
& \sqrt{\lambda} \arctan \frac{1}{\sqrt{\lambda}} .
\end{aligned}
$$

The first family of complete Bernstein functions corresponds to $\alpha$-stable subordinators when $\alpha \in(0,1)$ and to the pure drift when $\alpha=1$, the second family to relativistic $\alpha$-stable subordinators, and the third to gamma subordinators. An example of a Bernstein function which is not a complete Bernstein function is $1-\mathrm{e}^{-\lambda}$.

Suppose that $T$ is a subordinator whose Laplace exponent is a complete Bernstein function. The following theorem shows that if the drift $b$ of $T$ is positive or the jumping measure $\nu$ of $T$ satisfies $\nu(0, \infty)=\infty$, the potential measure of $T$ is absolutely continuous with respect to the Lebesgue measure and the potential kernel is completely monotone. The theorem also gives an explicit formula for the potential kernel. For results related to this theorem, see Section 14 of [5].

THEOREM 2.3. Suppose that $T=\left(T_{t}: t \geqslant 0\right)$ is a subordinator whose Laplace exponent

$$
\phi(\lambda)=b \lambda+\int_{0}^{\infty}\left(1-e^{-\lambda t}\right) \nu(d t)
$$

is a complete Bernstein function. Assume that $b>0$ or $\nu(0, \infty)=\infty$. Then the potential measure $U$ has a density $u$ which is completely monotone on $(0, \infty)$.

Proof. Since $\phi$ is a complete Bernstein function, $\tilde{\phi}(\lambda):=\lambda / \phi(\lambda)$ is also a complete Bernstein function ([13], pp. 193-194), so there is a Bernstein function $\xi$ such that

$$
\frac{\lambda}{\phi(\lambda)}=\tilde{\phi}(\lambda)=\lambda^{2} \mathcal{L} \xi(\lambda) .
$$


Therefore we have

$$
\frac{1}{\phi(\lambda)}=\lambda \mathcal{L} \xi(\lambda)
$$

It follows easily that

$$
\lim _{\lambda \rightarrow 0} \tilde{\phi}(\lambda)=\lim _{\lambda \rightarrow 0} \frac{\lambda}{\phi(\lambda)}=\frac{1}{b+\int_{0}^{\infty} t \nu(\mathrm{d} t)} .
$$

Also, since $b>0$ or $\nu(0, \infty)=\infty$, we have that

$$
\lim _{\lambda \rightarrow \infty} \frac{\tilde{\phi}(\lambda)}{\lambda}=\lim _{\lambda \rightarrow \infty} \frac{1}{\phi(\lambda)}=0 .
$$

Therefore, $\tilde{\phi}$ must have a representation

$$
\tilde{\phi}(\lambda)=\frac{1}{b+\int_{0}^{\infty} t \nu(\mathrm{d} t)}+\int_{0}^{\infty}\left(1-\mathrm{e}^{-\lambda t}\right) \mu(\mathrm{d} t)
$$

for some Lévy measure $\mu$ on $(0, \infty)$. It follows from Lemma 2.2 that the Bernstein function $\xi$ must have the representation

$$
\xi(\lambda)=\frac{\lambda}{b+\int_{0}^{\infty} t \nu(\mathrm{d} t)}+\int_{0}^{\infty}\left(1-\mathrm{e}^{-\lambda t}\right) \gamma(\mathrm{d} t)
$$

for some Lévy measure $\gamma$ on $(0, \infty)$. Consequently $\xi(0+)=0$, and from (2.7),

$$
\frac{1}{\phi(\lambda)}=\left(\mathcal{L} \xi^{\prime}\right)(\lambda)
$$

Together with (2.6) this gives

$$
\mathcal{L} U(\lambda)=\mathcal{L} \xi^{\prime}(\lambda)
$$

This equality of Laplace transforms implies that we can take $u(x):=\xi^{\prime}(x), x>0$, as a density of the potential measure $U$. Since $\xi$ is a Bernstein function, $\xi^{\prime}$ is completely monotone.

\section{Green Function Estimates}

Let $Y=\left(Y_{t}, t \geqslant 0\right)$ be a $d$-dimensional Brownian motion, $d \geqslant 3$, with the transition density given by

$$
p(t, x, y)=(4 \pi t)^{-d / 2} \exp \left(-\frac{|x-y|^{2}}{4 t}\right), x, y \in \mathbb{R}^{d}, t>0 .
$$


The Green function of $Y$ is

$$
G^{(2)}(x, y)=\frac{\Gamma(d / 2-1)}{4 \pi^{d / 2}}|x-y|^{2-d} .
$$

Let $T=\left(T_{t}: t \geqslant 0\right)$ be a subordinator independent of the Brownian motion $Y$, and let

$$
\phi(\lambda)=b \lambda+\int_{0}^{\infty}\left(1-\mathrm{e}^{-\lambda t}\right) \nu(\mathrm{d} t)
$$

be the Laplace exponent of $T$. We assume that $T$ is not a compound Poisson process. Denote by $\mu_{t}$ the distribution of $T_{t}: \mu_{t}(\mathrm{~d} s):=\mathbb{P}\left(T_{t} \in \mathrm{d} s\right)$. The process $X$ subordinate to $Y$ by the subordinator $T$ is defined as $X_{t}:=Y\left(T_{t}\right), t \geqslant 0$. We call $X$ a subordinate Brownian motion.

The transition density of $X$ is

$$
q(t, x, y):=\int_{0}^{\infty} p(s, x, y) \mu_{t}(\mathrm{~d} s)
$$

The potential operator $G f(x):=\mathbb{E}^{x} \int_{0}^{\infty} f\left(X_{t}\right) \mathrm{d} t$ of $X$ has a density given by

$$
G(x, y):=\int_{0}^{\infty} q(t, x, y) \mathrm{d} t .
$$

We call $G$ the Green function of $X$. When the potential measure $U$ of the subordinator $T$ has a density $u$, we have

$$
\begin{aligned}
G(x, y) & =\int_{0}^{\infty} \int_{0}^{\infty} p(s, x, y) \mu_{t}(\mathrm{~d} s) \mathrm{d} t \\
& =\int_{0}^{\infty} p(s, x, y) U(\mathrm{~d} s) \\
& =\int_{0}^{\infty} p(s, x, y) u(s) \mathrm{d} s .
\end{aligned}
$$

In the rest of the paper the asymptotic behavior of the Laplace exponent at infinity will play a crucial role. Note that if the drift $b$ of the subordinator $T$ is strictly positive, then $\phi(\lambda) \sim b \lambda$ as $\lambda \rightarrow \infty$. Conversely, if $\phi(\lambda) \sim b \lambda$ as $\lambda \rightarrow \infty$ for some $b>0$, then $b$ is the drift of the subordinator. We will be also interested in the case when the Laplace exponent of $T$ is a complete Bernstein function satisfying $\phi(\lambda) \sim \gamma^{-1} \lambda^{\alpha / 2}$ as $\lambda \rightarrow \infty$ for $\alpha \in(0,2)$ and a positive constant $\gamma$. 
Note that in this case the drift of the subordinator must be zero. We will put these two cases into the following assumption:

$$
\phi(\lambda) \sim \gamma^{-1} \lambda^{\alpha / 2}, \lambda \rightarrow \infty, \alpha \in(0,2],
$$

where in the case $\alpha \in(0,2)$ we always assume that the Laplace exponent is a complete Bernstein function. Note that in the case $\alpha=2$, we have that $\gamma^{-1}=b$, the drift of the subordinator.

THEOREM 3.1. If the Laplace exponent of the subordinator $T$ satisfies the assumption (3.3), then

$$
G(x, y) \sim \frac{\gamma}{\pi^{d / 2} 2^{\alpha}} \frac{\Gamma\left(\frac{d-\alpha}{2}\right)}{\Gamma\left(\frac{\alpha}{2}\right)}|x-y|^{\alpha-d}
$$

as $|x-y| \rightarrow 0$.

Proof. Case $\alpha=2$. Since $b>0$, the potential measure $U$ has a density $u$. Note first that by change of variables

$$
\int_{0}^{\infty}(4 \pi t)^{-d / 2} \exp \left(-\frac{|x|^{2}}{4 t}\right) u(t) \mathrm{d} t=\frac{|x|^{-d+2}}{4 \pi^{d / 2}} \int_{0}^{\infty} s^{d / 2-2} \mathrm{e}^{-s} u\left(\frac{|x|^{2}}{4 s}\right) \mathrm{d} s .
$$

By Lemma 2.1, $\lim _{x \rightarrow 0} u\left(|x|^{2} /(4 s)\right)=u(0+)=1 / b$ for all $s>0$ and the convergence is bounded by $u(0+)$. Hence, by the bounded convergence theorem,

$$
\lim _{x \rightarrow 0} \frac{1}{|x|^{-d+2}} \int_{0}^{\infty}(4 \pi t)^{-d / 2} \exp \left(-\frac{|x|^{2}}{4 t}\right) u(t) \mathrm{d} t=\frac{\Gamma(d / 2-1)}{4 \pi^{d / 2} b} .
$$

Case $0<\alpha<2$. Note that by Theorem 2.3 the potential measure of the subordinator has a decreasing density. By use of the Tauberian theorem and monotone density theorem (e.g. [7]), the assumption $\phi(\lambda) \sim \gamma^{-1} \lambda^{\alpha / 2}$ as $\lambda \rightarrow \infty$ implies that

$$
u(t) \sim \frac{\gamma}{\Gamma(\alpha / 2)} t^{\alpha / 2-1}, \quad t \rightarrow 0+.
$$

Hence, there is a constant $c>0$ such that

$$
\frac{u(t)}{\frac{\gamma}{\Gamma(\alpha / 2)} t^{\alpha / 2-1}} \leqslant \begin{cases}c, & t<1 \\ c t^{-\alpha / 2+1}, & t \geqslant 1\end{cases}
$$


By use of (3.4) we have

$$
\begin{aligned}
\int_{0}^{\infty} & (4 \pi t)^{-d / 2} \exp \left(-\frac{|x|^{2}}{4 t}\right) u(t) \mathrm{d} t \\
& =\frac{|x|^{-d+2}}{4 \pi^{d / 2}} \int_{0}^{\infty} s^{d / 2-2} \mathrm{e}^{-s} u\left(\frac{|x|^{2}}{4 s}\right) \mathrm{d} s \\
& =\frac{\gamma}{4 \pi^{d / 2} \Gamma(\alpha / 2)}|x|^{-d+\alpha} \int_{0}^{\infty} s^{d / 2-2} \mathrm{e}^{-s} \frac{u\left(\frac{|x|^{2}}{4 s}\right)}{\frac{\gamma}{\Gamma(\alpha / 2)}\left(\frac{|x|^{2}}{4 s}\right)^{\alpha / 2-1}}\left(\frac{1}{4 s}\right)^{\alpha / 2-1} \mathrm{~d} s \\
& =\frac{\gamma}{2^{\alpha} \pi^{d / 2} \Gamma(\alpha / 2)}|x|^{-d+\alpha} \int_{0}^{\infty} s^{d / 2-\alpha / 2-1} \mathrm{e}^{-s} \frac{u\left(\frac{|x|^{2}}{4 s}\right)}{\frac{\gamma}{\Gamma(\alpha / 2)}\left(\frac{|x|^{2}}{4 s}\right)^{\alpha / 2-1}} \mathrm{~d} s \\
& =\frac{\gamma}{2^{\alpha} \pi^{d / 2} \Gamma(\alpha / 2)}|x|^{-d+\alpha}\left(I_{1}+I_{2}\right),
\end{aligned}
$$

where

$$
\begin{aligned}
& I_{1}=\int_{0}^{|x|^{2} / 4} s^{d / 2-\alpha / 2-1} \mathrm{e}^{-s} \frac{u\left(\frac{|x|^{2}}{4 s}\right)}{\frac{\gamma}{\Gamma(\alpha / 2)}\left(\frac{|x|^{2}}{4 s}\right)^{\alpha / 2-1}} \mathrm{~d} s, \\
& I_{2}=\int_{|x|^{2} / 4}^{\infty} s^{d / 2-\alpha / 2-1} \mathrm{e}^{-s} \frac{u\left(\frac{|x|^{2}}{4 s}\right)}{\frac{\gamma}{\Gamma(\alpha / 2)}\left(\frac{|x|^{2}}{4 s}\right)^{\alpha / 2-1}} \mathrm{~d} s .
\end{aligned}
$$

In the second integral $I_{2}$, the integrand is bounded by the integrable function $s \rightarrow c s^{d / 2-\alpha / 2-1} \mathrm{e}^{-s}$, hence by the dominated convergence theorem and (3.6),

$$
\lim _{|x| \rightarrow 0} I_{2}=\int_{0}^{\infty} s^{d / 2-\alpha / 2-1} \mathrm{e}^{-s} \mathrm{~d} s=\Gamma\left(\frac{d-\alpha}{2}\right) .
$$

For the first integral we have

$$
\begin{aligned}
I_{1} & \leqslant \int_{0}^{|x|^{2} / 4} s^{d / 2-\alpha / 2-1} \mathrm{e}^{-s} c\left(\frac{|x|^{2}}{4 s}\right)^{-\alpha / 2+1} \mathrm{~d} s \\
& =c 4^{\alpha / 2-1}|x|^{-\alpha+2} \int_{0}^{|x|^{2} / 4} s^{d / 2-2} \mathrm{e}^{-s} \mathrm{~d} s .
\end{aligned}
$$


As $|x| \rightarrow 0$, the right-hand side above also converges to zero. Hence, $\lim _{|x| \rightarrow 0}$ $I_{1}=0$.

Therefore,

$$
\begin{aligned}
\lim _{|x| \rightarrow 0} \frac{1}{|x|^{\alpha-d}} \int_{0}^{\infty}(4 \pi t)^{-d / 2} \exp \left(-\frac{|x|^{2}}{4 t}\right) u(t) \mathrm{d} t & =\lim _{|x| \rightarrow 0} \frac{\gamma}{2^{\alpha} \pi^{d / 2} \Gamma(\alpha / 2)}\left(I_{1}+I_{2}\right) \\
& =\frac{\gamma}{2^{\alpha} \pi^{d / 2}} \frac{\Gamma\left(\frac{d-\alpha}{2}\right)}{\Gamma\left(\frac{\alpha}{2}\right)} .
\end{aligned}
$$

Remark 3.2. Recall that the Green function $G^{(\alpha)}$ of the rotationally invariant $\alpha$-stable process in $\mathbb{R}^{d}$ has the form

$$
G^{(\alpha)}(x, y)=\frac{1}{\pi^{d / 2} 2^{\alpha}} \frac{\Gamma\left(\frac{d-\alpha}{2}\right)}{\Gamma\left(\frac{\alpha}{2}\right)}|x-y|^{\alpha-d}, \quad x, y \in \mathbb{R}^{d},
$$

with $\alpha \in(0,2]$. It follows from the theorem above that there exist two positive constants, $c_{1}$ and $c_{2}$ (depending on $\gamma$ ), such that for all $x, y \in \mathbb{R}^{d}$ satisfying $|x-y| \leqslant 2$,

$$
c_{1} G^{(\alpha)}(x, y) \leqslant G(x, y) \leqslant c_{2} G^{(\alpha)}(x, y) .
$$

We look now at the asymptotic behavior of the Green function $G(x, y)$ for $|x-y| \rightarrow \infty$.

THEOREM 3.3. Suppose that $T=\left(T_{t}: t \geqslant 0\right)$ is a subordinator whose Laplace exponent

$$
\phi(\lambda)=b \lambda+\int_{0}^{\infty}\left(1-\mathrm{e}^{-\lambda t}\right) \nu(\mathrm{d} t)
$$

is a complete Bernstein function. If $\phi(\lambda) \sim \gamma^{-1} \lambda^{\alpha / 2}$ as $\lambda \rightarrow 0+$ for $\alpha \in(0,2]$ and a positive constant $\gamma$, then

$$
G(x, y) \sim \frac{\gamma}{\pi^{d / 2} 2^{\alpha}} \frac{\Gamma\left(\frac{d-\alpha}{2}\right)}{\Gamma\left(\frac{\alpha}{2}\right)}|x-y|^{\alpha-d}
$$

as $|x-y| \rightarrow \infty$.

Proof. Note that the assumption that $\phi(\lambda) \sim \gamma^{-1} \lambda^{\alpha / 2}$ as $\lambda \rightarrow 0+$ implies that either $b>0$ or $\nu(0, \infty)=\infty$. Thus by Theorem 2.3 the potential measure of the subordinator has a decreasing density. By use of the Tauberian theorem and 
monotone density theorem (e.g., [7]), the assumption $\phi(\lambda) \sim \gamma^{-1} \lambda^{\alpha / 2}$ as $\lambda \rightarrow$ $0+$ implies that

$$
u(t) \sim \frac{\gamma}{\Gamma(\alpha / 2)} t^{\alpha / 2-1}, \quad t \rightarrow \infty .
$$

Since $u$ is decreasing and integrable near 0 , it is easy to show that there exists $t_{0}>0$ such that $u(t) \leqslant t^{-1}$ for all $t \in\left(0, t_{0}\right)$. Hence, we can find a positive constant $C$ such that

$$
u(t) \leqslant C\left(t^{-1} \vee t^{\alpha / 2-1}\right) .
$$

By use of (3.4) we have

$$
\begin{aligned}
\int_{0}^{\infty} & (4 \pi t)^{-d / 2} \exp \left(-\frac{|x|^{2}}{4 t}\right) u(t) \mathrm{d} t \\
& =\frac{1}{4 \pi^{d / 2}}|x|^{-d+2} \int_{0}^{\infty} s^{d / 2-2} \mathrm{e}^{-s} u\left(\frac{|x|^{2}}{4 s}\right) \mathrm{d} s \\
& =\frac{\gamma}{4 \pi^{d / 2} \Gamma(\alpha / 2)}|x|^{-d+\alpha} \int_{0}^{\infty} s^{d / 2-2} \mathrm{e}^{-s} \frac{u\left(\frac{|x|^{2}}{4 s}\right)}{\frac{\gamma}{\Gamma(\alpha / 2)}\left(\frac{|x|^{2}}{4 s}\right)^{\alpha / 2-1}}\left(\frac{1}{4 s}\right)^{\alpha / 2-1} \mathrm{~d} s \\
& \left.=\frac{\gamma}{2^{\alpha} \pi^{d / 2} \Gamma(\alpha / 2)}|x|^{-d+\alpha} \int_{0}^{\infty} s^{d / 2-\alpha / 2-1} \mathrm{e}^{-s} \frac{\left.\frac{\gamma}{4 s}\right)}{\frac{|x|^{2}}{4(\alpha / 2)}}\right)^{\alpha / 2-1} \mathrm{~d} s
\end{aligned}
$$

Let $|x| \geqslant 2$. Then by (3.8),

$$
\begin{aligned}
\frac{u\left(\frac{|x|^{2}}{4 s}\right)}{\left(\frac{|x|^{2}}{4 s}\right)^{\alpha / 2-1}} & \leqslant C\left(\left(\frac{|x|^{2}}{4 s}\right)^{-\alpha / 2} \vee 1\right) \\
& \leqslant C\left(s^{\alpha / 2} \vee 1\right) .
\end{aligned}
$$

It follows that the integrand in the last formula above is bounded by an integrable function, so we may use the bounded convergence theorem to obtain

$$
\lim _{|x| \rightarrow \infty} \frac{1}{|x|^{-d+\alpha}} \int_{0}^{\infty}(4 \pi t)^{-d / 2} \exp \left(-\frac{|x|^{2}}{4 t}\right) u(t) d t=\frac{\gamma}{2^{\alpha} \pi^{d / 2}} \frac{\Gamma\left(\frac{d-\alpha}{2}\right)}{\Gamma\left(\frac{\alpha}{2}\right)},
$$

which proves the result.

Typical examples that satisfy the assumptions of the last theorem are subordinators of the form $T_{t}=b t+\tilde{T}_{t}$ where $b$ is nonnegative, $\tilde{T}$ is a $\beta / 2$-stable subordinator, or a relativistic $\beta / 2$-stable subordinator, or a gamma subordinator. 
In the first case the $\alpha$ in the theorem above is equal to $\beta$, while in the last two cases we have $\alpha=2$. In fact, whenever $\tilde{T}$ is a subordinator with finite expectation and such that the Laplace exponent of $T$ is a complete Bernstein function, the proposition will hold true with $\alpha=2$ and $\gamma^{-1}=\phi^{\prime}(0+)$.

Remark 3.4. Suppose that $T_{t}=b t+\tilde{T}_{t}$ where $b$ is positive and $\tilde{T}_{t}$ is a pure jump subordinator with finite expectation and the Laplace exponent that is a complete Bernstein function. Then $\phi(\lambda) \sim b \lambda, \lambda \rightarrow \infty$, and $\phi(\lambda) \sim \phi^{\prime}(0+) \lambda$, $\lambda \rightarrow 0$. This implies that the Green function of the subordinate process $X$ satisfies $G(x, y) \asymp G^{(2)}(x, y)$ for all $x, y \in \mathbb{R}^{d}$.

For any Borel subset $A$ of $\mathbb{R}^{d}$, we use $\sigma_{A}$ to denote the first hitting time of $A$ :

$$
\sigma_{A}=\inf \left\{t>0: X_{t} \in A\right\} .
$$

For any open set $D$ in $\mathbb{R}^{d}$, we use $\tau_{D}$ to denote the first exit time from $D$ :

$$
\tau_{D}=\inf \left\{t>0: X_{t} \notin D\right\} .
$$

In the remaining part of the paper we fix a constant $c$ such that

$$
c>\left(\frac{c_{2}}{c_{1}}\right)^{\frac{1}{d-\alpha}},
$$

where $c_{1}$ and $c_{2}$ are constants from (3.7). Note that $c>1$.

PROPOSITION 3.5. Assume that the Laplace exponent of the subordinator $T$ satisfies the assumption (3.3). Let $c>0$ be as in (3.10). Then there exist positive constants $c_{3}$ and $c_{4}$ such that for every $r \in(0,1)$

$$
c_{3} r^{\alpha} \leqslant \inf _{z \in B\left(0,(7 c)^{-1} r\right)} \mathbb{E}_{z} \tau_{B(0, r)} \leqslant \sup _{z \in B(0, r)} \mathbb{E}_{z} \tau_{B(0, r)} \leqslant c_{4} r^{\alpha}
$$

Proof. Recall that $\mathbb{E}_{z} \tau_{B(0, r)}=\int_{B(0, r)} G_{B(0, r)}(z, y) \mathrm{d} y$, where $G_{B(0, r)}$ is the Green function of the process $X$ killed upon exiting the ball $B(0, r)$ and it is given by $G_{B(0, r)}(z, y)=G(z, y)-\mathbb{E}_{z} G\left(X\left(\tau_{B(0, r)}\right), y\right)$, for $y, z \in B(0, r)$.

The right-hand side estimate in (3.11) follows immediately from the estimate (3.7) and the fact that $G_{B(0, r)}(z, y) \leqslant G(z, y)$. For the left-hand side estimate, let $y, z \in B\left(0,(7 c)^{-1} r\right)$. Then $|z-y| \leqslant 2(7 c)^{-1} r$ and $\left|X\left(\tau_{B(0, r)}\right)-y\right| \geqslant r-(7 c)^{-1}$ $r \geqslant r / 2$ (since $c>1$ ). By use of the estimate (3.7) and the fact that the radial part of $G$ is decreasing, we get

$$
\begin{aligned}
G_{B(0, r)}(z, y) & \geqslant c_{1}\left(2(7 c)^{-1} r\right)^{\alpha-d}-c_{2}(r / 2)^{\alpha-d} \\
& \geqslant r^{\alpha-d}\left(c_{1}(7 / 2)^{d-\alpha}\left(c_{2} / c_{1}\right)-c_{2} 2^{d-\alpha}\right) \\
& =c_{2} r^{\alpha-d}\left((7 / 2)^{d-\alpha}-2^{d-\alpha}\right) .
\end{aligned}
$$


Now for $z \in B\left(0,(7 c)^{-1}\right)$ we have

$$
\begin{aligned}
\mathbb{E}_{z} \tau_{B(0, r)} & =\int_{B(0, r)} G_{B(0, r)}(z, y) \mathrm{d} y \\
& \geqslant \int_{B\left(0,(7 c)^{-1} r\right)} G_{B(0, r)}(z, y) \mathrm{d} y \\
& \geqslant c_{2} r^{\alpha-d}\left((7 / 2)^{d-\alpha}-2^{d-\alpha}\right)|B(0, r)| \\
& =c_{3} r^{\alpha} .
\end{aligned}
$$

Remark 3.6. By use of a different argument, it is proved in [25] that under more general assumptions on the underlying process there are positive constants $c_{3}$ and $c_{4}$ such that

$$
c_{3} r^{\alpha} \leqslant \inf _{z \in B(0, r / 2)} \mathbb{E}_{z} \tau_{B(0, r)} \leqslant \sup _{z \in B(0, r)} \mathbb{E}_{z} \tau_{B(0, r)} \leqslant c_{4} r^{\alpha}
$$

In the sequel we will use $\operatorname{Cap}(A)$ to denote the 0 -capacity of the set $A$ with respect to $X$ and $\operatorname{Cap}^{(\alpha)}(A)$ to denote the 0 -capacity of the set $A$ with respect to the rotationally invariant $\alpha$-stable process.

PROPOSITION 3.7. Assume that the Laplace exponent of the subordinator $T$ satisfies the assumption (3.3). Then for any Borel subset $A \subset B(0,1)$,

$$
c_{2}^{-1} \operatorname{Cap}^{(\alpha)}(A) \leqslant \operatorname{Cap}(A) \leqslant c_{1}^{-1} \operatorname{Cap}^{(\alpha)}(A),
$$

with constants $c_{1}$ and $c_{2}$ from the estimate (3.7).

Proof. It is well known that

$$
\operatorname{Cap}(A)=\sup \left\{\mu(A): \operatorname{supp}(\mu) \subset \bar{A} \text { and } G \mu \leqslant 1 \text { a.e. on } \mathbb{R}^{d}\right\} \text {. }
$$

Using Remark 3.2 we get that if $\mu$ is a measure supported in $\bar{A}$ with $G \mu \leqslant 1$ almost everywhere on $\mathbb{R}^{d}$, then the measure $\nu:=c_{1} \mu$ is a measure supported in $\bar{A}$ with $G^{(\alpha)} \nu \leqslant 1$ almost everywhere on $\mathbb{R}^{d}$. Here we also used the maximum principle for the potential of measures. Thus we have

$$
\begin{aligned}
\operatorname{Cap}(A) & =\sup \left\{\mu(A): \operatorname{supp}(\mu) \subset \bar{A} \text { and } G \mu \leqslant 1 \text { a.e. on } \mathbb{R}^{d}\right\} \\
& =c_{1}^{-1} \sup \left\{c_{1} \mu(A): \operatorname{supp}(\mu) \subset \bar{A} \text { and } G \mu \leqslant 1 \text { a.e. on } \mathbb{R}^{d}\right\} \\
& \leqslant c_{1}^{-1} \sup \left\{\nu(A): \operatorname{supp}(\nu) \subset \bar{A} \text { and } G^{(\alpha)} \nu \leqslant 1 \text { a.e. on } \mathbb{R}^{d}\right\} \\
& =c_{1}^{-1} \operatorname{Cap}^{(\alpha)}(A) .
\end{aligned}
$$

The left-hand side inequality is proved in the same way. 


\section{Harnack Inequality}

In this section we prove the Harnack inequality for the nonnegative harmonic functions of $X$. The first necessary ingredient is the lower bound on the probability of $X$ hitting sets of positive capacity before exiting a small ball.

LEMMA 4.1. Assume that the Laplace exponent of the subordinator T satisfies the assumption (3.3). Let $c>0$ be as in (3.10). Then there exist positive constants $c_{5}$ and $c_{6}$ such that for any $r \in\left(0,(7 c)^{-1}\right)$, any closed subset $A$ of $B(0, r)$ and any $y \in B(0, r)$,

$$
\begin{aligned}
& \mathbb{P}_{y}\left(\sigma_{A}<\tau_{B(0,7 c r)}\right) \geqslant c_{5} \frac{\operatorname{Cap}^{(\alpha)}(A)}{\operatorname{Cap}^{(\alpha)}(B(0, r))}, \\
& \mathbb{P}_{y}\left(\sigma_{A}<\tau_{B(0,7 c r)}\right) \geqslant c_{6} \frac{\operatorname{Cap}(A)}{\operatorname{Cap}(B(0, r))} .
\end{aligned}
$$

Proof. It follows from Proposition 3.7 that it is enough to prove one of the inequalities above. We will only prove the first inequality.

Without loss of generality we may assume that $\operatorname{Cap}^{(\alpha)}(A)>0$. Let $G_{B(0,7 c r)}$ be the Green function of the process obtained by killing $X$ upon exiting from $B(0,7 \mathrm{cr})$. If $\nu$ is the capacitary measure of $A$ with respect to $X$, then we have for all $y \in B(0, r)$,

$$
\begin{aligned}
G_{B(0,7 c r)} \nu(y) & =\mathbb{E}_{y}\left[G_{B(0,7 c r)} \nu\left(X_{\sigma_{A}}\right): \sigma_{A}<\tau_{B(0,7 c r)}\right] \\
& \leqslant \sup _{z \in \mathbb{R}^{d}} G_{B(0,7 c r)} \nu(z) \mathbb{P}_{y}\left(\sigma_{A}<\tau_{B(0,7 c r)}\right) \\
& \leqslant \mathbb{P}_{y}\left(\sigma_{A}<\tau_{B(0,7 c r)}\right) .
\end{aligned}
$$

On the other hand, we have for all $y \in B(0, r)$,

$$
\begin{aligned}
G_{B(0,7 c r)} \nu(y) & =\int G_{B(0,7 c r)}(y, z) \nu(d z) \geqslant \nu(A) \inf _{z \in B(0, r)} G_{B(0,7 c r)}(y, z) \\
& =\operatorname{Cap}(A) \inf _{z \in B(0, r)} G_{B(0,7 c r)}(y, z) \\
& \geqslant c_{2}^{-1} \operatorname{Cap}^{(\alpha)}(A) \inf _{z \in B(0, r)} G_{B(0,7 c r)}(y, z) .
\end{aligned}
$$

Since $G_{B(0,7 c r)}(y, z)=G(y, z)-\mathbb{E}_{y}\left[G\left(X_{\tau_{B(0,7 c r)}}, z\right)\right]$, we have for all $y, z \in B(0, r)$,

$$
\begin{aligned}
G_{B(0,7 c r)}(y, z) & \geqslant c_{1} G^{(\alpha)}(y, z)-c_{2} \mathbb{E}_{y}\left[G^{(\alpha)}\left(X_{\tau_{B(0,7 c r)}}, z\right)\right] \\
& \geqslant c_{1}(2 r)^{\alpha-d}-c_{2}(6 c r)^{\alpha-d} \geqslant c_{7} r^{\alpha-d}=\frac{c_{8}}{\operatorname{Cap}^{(\alpha)}(B(0, r))} .
\end{aligned}
$$


In the first inequality above we used the estimate (3.7) and the fact that the radial part of $G$ is decreasing. Therefore

$$
\mathbb{P}_{y}\left(\sigma_{A}<\tau_{B(0,7 c r)}\right) \geqslant c_{5} \frac{\operatorname{Cap}^{(\alpha)}(A)}{\operatorname{Cap}^{(\alpha)}(B(0, r))} .
$$

The Lévy measure of the subordinate Brownian motion $X$ has a density given by the formula

$$
\iota(x)=\int_{0}^{\infty}(4 \pi t)^{-d / 2} \exp \left(-\frac{|x|^{2}}{4 t}\right) \nu(\mathrm{d} t) .
$$

(see, for example, [19], pp. 197-198). Here $\nu$ is the Lévy measure of the subordinator. In this section we will assume that the Lévy measure has a density: $\nu(d t)=\nu(t) d t$. In order to study the properties of the Lévy measure of $X$, we introduce the following function $j:[0, \infty) \rightarrow[0, \infty)$ :

$$
j(u):=\int_{0}^{\infty} t^{-d / 2} \exp \left(-\frac{u^{2}}{4 t}\right) \nu(t) \mathrm{d} t, \quad u>0 .
$$

Note that $\iota(x)=(4 \pi)^{-d / 2} j(|x|)$ for all $x \in \mathbb{R}^{d}$. In the following, rather technical, lemma we give conditions on the density $\nu$ that imply properties of $j$ described as (3.3) and (3.4) in [24].

LEMMA 4.2. Suppose that there exists a positive constant $c_{9}>0$ such that

$$
\begin{aligned}
& \nu(t) \leqslant c_{9} \nu(2 t) \text { for all } t \in(0,8) \\
& \nu(t) \leqslant c_{9} \nu(t+1) \quad \text { for all } t>1 .
\end{aligned}
$$

Then there exists a positive constant $c_{10}$ such that

$$
\begin{aligned}
& j(u) \leqslant c_{10} j(2 u) \quad \text { for all } u \in(0,2), \\
& j(u) \leqslant c_{10} j(u+1) \text { for all } u>1 .
\end{aligned}
$$

Also, $u \mapsto j(u)$ is decreasing on $(0, \infty)$. 
Proof. Let $0<u<2$. We have

$$
\begin{aligned}
j(2 u)= & \int_{0}^{\infty} t^{-d / 2} \exp \left(-u^{2} / t\right) \nu(t) \mathrm{d} t \\
= & \frac{1}{2}\left(\int_{0}^{1 / 2} t^{-d / 2} \exp \left(-u^{2} / t\right) \nu(t) \mathrm{d} t+\int_{1 / 2}^{\infty} t^{-d / 2} \exp \left(-u^{2} / t\right) \nu(t) \mathrm{d} t\right. \\
& \left.+\int_{0}^{2} t^{-d / 2} \exp \left(-u^{2} / t\right) \nu(t) \mathrm{d} t+\int_{2}^{\infty} t^{-d / 2} \exp \left(-u^{2} / t\right) \nu(t) \mathrm{d} t\right) \\
\geqslant & \frac{1}{2}\left(\int_{1 / 2}^{\infty} t^{-d / 2} \exp \left(-u^{2} / t\right) \nu(t) \mathrm{d} t+\int_{0}^{2} t^{-d / 2} \exp \left(-u^{2} / t\right) \nu(t) \mathrm{d} t\right) \\
= & \frac{1}{2}\left(I_{1}+I_{2}\right) .
\end{aligned}
$$

Now,

$$
\begin{aligned}
I_{1} & =\int_{1 / 2}^{\infty} t^{-d / 2} \exp \left(-u^{2} / t\right) \nu(t) \mathrm{d} t \\
& =\int_{1 / 2}^{\infty} t^{-d / 2} \exp \left(-\frac{u^{2}}{4 t}\right) \exp \left(-\frac{3 u^{2}}{4 t}\right) \nu(t) \mathrm{d} t \\
& \geqslant \int_{1 / 2}^{\infty} t^{-d / 2} \exp \left(-\frac{u^{2}}{4 t}\right) \exp \left(-\frac{3 u^{2}}{2}\right) \nu(t) \mathrm{d} t \\
& \geqslant \mathrm{e}^{-6} \int_{1 / 2}^{\infty} t^{-d / 2} \exp \left(-\frac{u^{2}}{4 t}\right) \nu(t) \mathrm{d} t \\
I_{2} & =\int_{0}^{2} t^{-d / 2} \exp \left(-u^{2} / t\right) \nu(t) \mathrm{d} t \\
& =4^{-d / 2+1} \int_{0}^{1 / 2} s^{-d / 2} \exp \left(-\frac{u^{2}}{4 s}\right) \nu(4 s) \mathrm{d} s \\
& \geqslant c_{9}^{-2} 4^{-d / 2+1} \int_{0}^{1 / 2} s^{-d / 2} \exp \left(-\frac{u^{2}}{4 s}\right) \nu(s) \mathrm{d} s .
\end{aligned}
$$


Combining the three terms above we get that

$$
j(2 u) \geqslant c_{11} j(u), \quad u \in(0,2) .
$$

To prove (4.6) we first note that for all $t \geqslant 2$ and all $u \geqslant 1$ it holds that

$$
\frac{(u+1)^{2}}{t}-\frac{u^{2}}{t-1} \leqslant 1
$$

This implies that

$$
\exp \left(-\frac{(u+1)^{2}}{4 t}\right) \geqslant \mathrm{e}^{-1 / 4} \exp \left(-\frac{u^{2}}{4(t-1)}\right), \quad \text { for all } u>1, t>2
$$

Now we have

$$
\begin{aligned}
j(u+1)= & \int_{0}^{\infty} t^{-d / 2} \exp \left(-\frac{(u+1)^{2}}{4 t}\right) \nu(t) \mathrm{d} t \\
\geqslant & \frac{1}{2}\left(\int_{0}^{8} t^{-d / 2} \exp \left(-\frac{(u+1)^{2}}{4 t}\right) \nu(t) \mathrm{d} t\right. \\
& \left.+\int_{3}^{\infty} t^{-d / 2} \exp \left(-\frac{(u+1)^{2}}{4 t}\right) \nu(t) \mathrm{d} t\right) \\
= & \frac{1}{2}\left(I_{3}+I_{4}\right) .
\end{aligned}
$$

For $I_{3}$ note that $(u+1)^{2} \leqslant 4 u^{2}$ for all $u>1$. Thus

$$
\begin{aligned}
I_{3} & =\int_{0}^{8} t^{-d / 2} \exp \left(-\frac{(u+1)^{2}}{4 t}\right) \nu(t) \mathrm{d} t \\
& \geqslant \int_{0}^{8} t^{-d / 2} \exp \left(-u^{2} / t\right) \nu(t) \mathrm{d} t \\
& =4^{-d / 2+1} \int_{0}^{2} s^{-d / 2} \exp \left(-\frac{u^{2}}{4 s}\right) \nu(4 s) \mathrm{d} s \\
& \geqslant c_{9}^{-2} 4^{-d / 2+1} \int_{0}^{2} s^{-d / 2} \exp \left(-\frac{u^{2}}{4 s}\right) \nu(s) \mathrm{d} s .
\end{aligned}
$$


Next,

$$
\begin{aligned}
I_{4} & =\int_{3}^{\infty} t^{-d / 2} \exp \left(-\frac{(u+1)^{2}}{4 t}\right) \nu(t) \mathrm{d} t \\
& \geqslant \int_{3}^{\infty} t^{-d / 2} \exp \{-1 / 4\} \exp \left(-\frac{u^{2}}{4(t-1)}\right) \nu(t) \mathrm{d} t \\
& =e^{-1 / 4} \int_{2}^{\infty}(s-1)^{-d / 2} \exp \left(-\frac{u^{2}}{4 s}\right) \nu(s+1) \mathrm{d} s \\
& \geqslant c_{9}^{-1} e^{-1 / 4} \int_{2}^{\infty} s^{-d / 2} \exp \left(-\frac{u^{2}}{4 s}\right) \nu(s) \mathrm{d} s .
\end{aligned}
$$

Combining the three terms above we get

$$
j(u+1) \geqslant c_{12} j(u), \quad u>1 .
$$

Remark 4.3. Suppose that $T_{t}=b t+\tilde{T}_{t}$, where $b$ is nonnegative and $\tilde{T}$ is an $\alpha / 2$-stable subordinator, or a gamma subordinator, or a relativistic $\alpha / 2$-stable subordinator. In the first case the Lévy measure is given by $\nu(t)=c(\alpha) t^{-1-\alpha / 2}$, in the second case by $\nu(t)=t^{-1} \mathrm{e}^{-t}$, and in the third case by $\nu(t)=$ $c(\alpha) \mathrm{e}^{-t} t^{-1-\alpha / 2}$ (see, for instance, [10] or [18]), where $c(\alpha)=(\alpha / 2) / \Gamma(1-\alpha / 2)$. It is straightforward to verify that in all three cases $\nu(t)$ satisfies (4.3) and (4.4).

Using the same argument as in the proof of Lemma 3.5 of [24], which is a modification of the original argument from [3], we can easily get the following result.

LEMMA 4.4. Suppose that the Lévy measure of the subordinator $T$ satisfies (4.3) and (4.4). Then there exist positive constants $c_{13}$ and $c_{14}$ such that if $r \in$ $(0,1), z \in B(0, r)$ and $H$ is a nonnegative function with support in $B(0,2 r)^{c}$, then

$$
\mathbb{E}_{z} H\left(X\left(\tau_{B(0, r)}\right)\right) \leqslant c_{13}\left(\mathbb{E}_{z} \tau_{B(0, r)}\right) \int H(y) j(|y|) \mathrm{d} y
$$

and

$$
\mathbb{E}_{z} H\left(X\left(\tau_{B(0, r)}\right)\right) \geqslant c_{14}\left(\mathbb{E}_{z} \tau_{B(0, r)}\right) \int H(y) j(|y|) \mathrm{d} y
$$

THEOREM 4.5. Assume that the Laplace exponent of the subordinator $T$ satisfies the assumption (3.3) and that the Lévy measure of the subordinator $T$ 
satisfies (4.3) and (4.4). Let $c>0$ be as in (3.10). Then there exists a constant $c_{15}>0$ such that, for any $r \in\left(0, \frac{1}{49 c}\right), x_{0} \in \mathbb{R}^{d}$ and any function $h$ which is nonnegative and bounded on $\mathbb{R}^{d}$, and harmonic with respect to $X$ in $B\left(x_{0}, 50 \mathrm{cr}\right)$, we have

$$
h(x) \leqslant c_{15} h(y), \quad x, y \in B\left(x_{0}, r\right) .
$$

Proof. This proof is based on the proof given in [3]. By looking at $h+\epsilon$ and letting $\epsilon \downarrow 0$, we may suppose that $h$ is bounded from below by a positive constant. By looking at $a h$ for a suitable $a>0$, we may suppose that $\inf _{B\left(x_{0}, r\right)} h=$ $1 / 2$. Choose $z_{0} \in B\left(x_{0}, r\right)$ such that $h\left(z_{0}\right) \leqslant 1$. We want to show that $h$ is bounded above in $B\left(x_{0}, r\right)$ by a constant $C>0$ independent of $h$ and $r \in\left(0, \frac{1}{49 c}\right)$. We will establish this by contradiction: If there exists a point $x \in B\left(x_{0}, r\right)$ with $h(x)=K$ where $K$ is larger than a constant to be determined later in the proof, we can obtain a sequence of points in $B\left(x_{0}, 2 r\right)$ along which $h$ is unbounded.

By Proposition 3.5 and Lemma 4.4, we can see that there exists $c_{16}>0$ such that if $x \in \mathbb{R}^{d}, s<r$, and $H$ is nonnegative bounded function with support in $B(x, 2 s)^{c}$, then for any $y, z \in B\left(x,(7 c)^{-1} s\right)$,

$$
\mathbb{E}_{z} H\left(X\left(\tau_{B(x, s)}\right)\right) \leqslant c_{16} \mathbb{E}_{y} H\left(X\left(\tau_{B(x, s)}\right)\right) .
$$

By Lemma 4.1, if $A \subset B\left(x_{0}, 7 r\right)$ then

$$
\mathbb{P}_{y}\left(\sigma_{A}<\tau_{B\left(x_{0}, 49 c r\right)}\right) \geqslant c_{5} \frac{\operatorname{Cap}^{(\alpha)}(A)}{\operatorname{Cap}^{(\alpha)}\left(B\left(x_{0}, 7 r\right)\right)}, \quad \forall y \in B\left(x_{0}, 7 r\right) .
$$

Again by Lemma 4.1, there exists $c_{17}>0$ such that if $x \in \mathbb{R}^{d}, s \in(0,1)$ and $F \subset B\left(x,(7 c)^{-1} s\right)$ with $\operatorname{Cap}^{(\alpha)}(F) / \operatorname{Cap}^{(\alpha)}\left(B\left(x,(7 c)^{-1} s\right)\right) \geqslant 1 / 3$, then

$$
\mathbb{P}_{x}\left(\sigma_{F}<\tau_{B(x, s)}\right) \geqslant c_{17}
$$

Let

$$
\eta=\frac{c_{17}}{3}, \quad \zeta=\left(\frac{1}{3} \wedge \frac{1}{c_{16}}\right) \eta .
$$

Now suppose there exists $x \in B\left(x_{0}, r\right)$ with $h(x)=K$ for

$$
K>\frac{2 \operatorname{Cap}^{(\alpha)}\left(B\left(x_{0}, 7 r\right)\right)}{c_{5} \zeta} \vee \frac{2(49 c)^{d-\alpha}}{c_{5} \zeta} .
$$

Let $s$ be chosen so that

$$
\operatorname{Cap}^{(\alpha)}\left(B\left(x,(7 c)^{-1} s\right)\right)=\frac{2 \operatorname{Cap}^{(\alpha)}\left(B\left(x_{0}, 7 r\right)\right)}{c_{5} \zeta K}<1 .
$$


Note that this implies

$$
s=49 c r\left(\frac{2}{c_{5} \zeta}\right)^{1 /(d-\alpha)} K^{-1 /(d-\alpha)}<r .
$$

Let us write $B_{s}$ for $B(x, s), \tau_{s}$ for $\tau_{B(x, s)}$, and similarly for $B_{2 s}$ and $\tau_{2 s}$. Let $A$ be a compact subset of

$$
A^{\prime}=\left\{u \in B\left(x,(7 c)^{-1} s\right): h(u) \geqslant \zeta K\right\} .
$$

It is well known that $h\left(X_{t}\right)$ is right continuous in $\left[0, \tau_{B\left(x_{0}, 49 c r\right)}\right)$. Since $z_{0} \in$ $B\left(x_{0}, r\right)$ and $A^{\prime} \subset B\left(x,(7 c)^{-1} s\right) \subset B\left(x_{0}, 2 r\right)$, we can apply (4.9) to get

$$
\begin{aligned}
1 & \geqslant h\left(z_{0}\right) \geqslant \mathbb{E}_{z_{0}}\left[h\left(X\left(\sigma_{A} \wedge \tau_{B\left(x_{0}, 49 c r\right)}\right)\right) 1_{\left\{\sigma_{A}<\tau_{B\left(x_{0}, 49 c r\right)}\right]}\right] \\
& \geqslant \zeta K \mathbb{P}_{z_{0}}\left[\sigma_{A}<\tau_{B\left(x_{0}, 49 c r\right)}\right] \\
& \geqslant c_{5} \zeta K \frac{\operatorname{Cap}^{(\alpha)}(A)}{\operatorname{Cap}^{(\alpha)}\left(B\left(x_{0}, 7 r\right)\right)} .
\end{aligned}
$$

Hence

$$
\frac{\operatorname{Cap}^{(\alpha)}(A)}{\operatorname{Cap}^{(\alpha)}\left(B\left(x,(7 c)^{-1} s\right)\right)} \leqslant \frac{\operatorname{Cap}^{(\alpha)}\left(B\left(x_{0}, 7 r\right)\right)}{c_{2} \zeta K \operatorname{Cap}^{(\alpha)}\left(B\left(x,(7 c)^{-1} s\right)\right)}=\frac{1}{2} .
$$

This implies that $\operatorname{Cap}^{(\alpha)}\left(A^{\prime}\right) / \operatorname{Cap}^{(\alpha)}\left(B\left(x,(7 c)^{-1} s\right)\right) \leqslant 1 / 2$. By subadditivity of Cap $^{(\alpha)}$ and capacitability of Borel sets, there exists $F$, a compact subset of $B\left(x,(7 c)^{-1} s\right) \backslash A^{\prime}$, such that

$$
\frac{\operatorname{Cap}^{(\alpha)}(F)}{\operatorname{Cap}^{(\alpha)}\left(B\left(x,(7 c)^{-1} s\right)\right)} \geqslant \frac{1}{3} .
$$

Let $H=h \cdot 1_{B_{2 s}^{c}}$. We claim that

$$
\mathbb{E}_{x}\left[h\left(X\left(\tau_{s}\right)\right) ; X\left(\tau_{s}\right) \notin B_{2 s}\right] \leqslant \eta K .
$$

If not, $\mathbb{E}_{x} H\left(X\left(\tau_{s}\right)\right)>\eta K$, and by (4.8), for all $y \in B\left(x,(7 c)^{-1} s\right)$, we have

$$
\begin{aligned}
h(y) & =\mathbb{E}_{y} h\left(X\left(\tau_{s}\right)\right) \geqslant \mathbb{E}_{y}\left[h\left(X\left(\tau_{s}\right)\right) ; X\left(\tau_{s}\right) \notin B_{2 s}\right] \\
& \geqslant c_{16}^{-1} \mathbb{E}_{x} H\left(X\left(\tau_{s}\right)\right) \geqslant c_{16}^{-1} \eta K \geqslant \zeta K,
\end{aligned}
$$

contradicting (4.14) and the definition of $A^{\prime}$. 
Let $M=\sup _{B_{2 s}} h$. We then have

$$
\begin{aligned}
K= & h(x)=\mathbb{E}_{x} h\left(X\left(\tau_{s}\right)\right) \\
= & \mathbb{E}_{x}\left[h\left(X\left(\sigma_{F}\right)\right) ; \sigma_{F}<\tau_{s}\right]+\mathbb{E}_{x}\left[h\left(X\left(\tau_{s}\right)\right) ; \tau_{s}<\sigma_{F} ; X\left(\tau_{s}\right) \in B_{2 s}\right] \\
& +\mathbb{E}_{x}\left[h\left(X\left(\tau_{s}\right)\right) ; \tau_{s}<\sigma_{F} ; X\left(\tau_{s}\right) \notin B_{2 s}\right] \\
& \leqslant \zeta K \mathbb{P}_{x}\left(\sigma_{F}<\tau_{s}\right)+M \mathbb{P}_{x}\left(\tau_{s}<\sigma_{F}\right)+\eta K \\
= & \zeta K \mathbb{P}_{x}\left(\sigma_{F}<\tau_{s}\right)+M\left(1-\mathbb{P}_{x}\left(\sigma_{F}<\tau_{s}\right)\right)+\eta K,
\end{aligned}
$$

or equivalently

$$
\frac{M}{K} \geqslant \frac{1-\eta-\zeta \mathbb{P}_{x}\left(\sigma_{F}<\tau_{s}\right)}{1-\mathbb{P}_{x}\left(\sigma_{F}<\tau_{s}\right)} .
$$

Using (4.10) and (4.11) we see that there exists $\beta>0$ such that $M \geqslant K(1+2 \beta)$. Therefore there exists $x^{\prime} \in B(x, 2 s)$ with $h\left(x^{\prime}\right) \geqslant K(1+\beta)$.

Now suppose there exists $x_{1} \in B\left(x_{0}, r\right)$ with $h\left(x_{1}\right)=K_{1}$. Define $s_{1}$ in terms of $K_{1}$ analogously to (4.12). Using the above argument (with $x_{1}$ replacing $x$ and $x_{2}$ replacing $\left.x^{\prime}\right)$, there exists $x_{2} \in B\left(x_{1}, 2 s_{1}\right)$ with $h\left(x_{2}\right)=K_{2} \geqslant(1+\beta) K_{1}$. We continue and obtain $s_{2}$ and then $x_{3}, K_{3}, s_{3}$, etc. Note that $x_{i+1} \in B\left(x_{i}, 2 s_{i}\right)$ and $K_{i} \geqslant(1+\beta)^{i-1} K_{1}$. In view of (4.13), $\sum_{i}\left|x_{i+1}-x_{i}\right| \leqslant c_{18} r K_{1}^{-1 /(d-\alpha)}$. So if $K_{1}>c_{18}^{d-\alpha}$, then we have a sequence $x_{1}, x_{2}, \ldots$ contained in $B\left(x_{0}, 2 r\right)$ with $h\left(x_{i}\right) \geqslant(1+\beta)^{i-1} K_{1} \rightarrow \infty$, a contradiction to $h$ being bounded. Therefore we can not take $K_{1}$ larger than $c_{18}^{d-\alpha}$, and thus $\sup _{y \in B\left(x_{0}, r\right)} h(y) \leqslant c_{18}^{d-\alpha}$, which is what we set out to prove.

By using standard chain argument, we can easily get the following consequence of the theorem above.

COROLLARY 4.6. For any domain $D$ of $\mathbb{R}^{d}$ and any compact subset $K$ of $D$, there exists a constant $c_{19}>0$ such that for any function $h$ which is nonnegative and bounded in $\mathbb{R}^{d}$ and harmonic with respect to $X$ in $D$, we have

$$
h(x) \leqslant c_{19} h(y), \quad x, y \in K .
$$

In the next result, we remove the boundedness assumption on the harmonic functions in Corollary 4.6.

THEOREM 4.7. For any domain $D$ of $\mathbb{R}^{d}$ and any compact subset $K$ of $D$, there exists a constant $c_{20}>0$ such that for any function $h$ which is nonnegative in $\mathbb{R}^{d}$ and harmonic with respect to $X$ in $D$, we have

$$
h(x) \leqslant c_{20} h(y), \quad x, y \in K .
$$


Proof. Choose a bounded domain $U$ such that $K \subset U \subset \bar{U} \subset D$. If $h$ is harmonic with respect to $X$ in $D$, then

$$
h(x)=\mathbb{E}_{x}\left[h\left(X\left(\tau_{U}\right)\right) 1_{\left\{\tau_{U}<\infty\right\}}\right], \quad x \in U .
$$

For any $n \geqslant 1$, define

$$
h_{n}(x)=\mathbb{E}_{x}\left[(h \wedge n)\left(X\left(\tau_{U}\right)\right) 1_{\left\{\tau_{U}<\infty\right\}}\right], \quad x \in \mathbb{R}^{d} .
$$

Then $h_{n}$ is a bounded nonnegative function on $\mathbb{R}^{d}$, harmonic with respect to $X$ in $U$, and

$$
\lim _{n \uparrow \infty} h_{n}(x)=h(x), \quad x \in \mathbb{R}^{d} .
$$

It follows from Corollary 4.6 that there exists a constant $c_{20}=c_{20}(U, K)>0$ such that

$$
h_{n}(x) \leqslant c_{20} h_{n}(y), \quad x, y \in K, n \geqslant 1 .
$$

Letting $n \uparrow \infty$, we get that

$$
h(x) \leqslant c_{20} h(y), \quad x, y \in K .
$$

\section{Extension to Subordinate Diffusions}

In this section we extend our results to subordinate diffusions. We will first consider elliptic diffusions on $\mathbb{R}^{d}, d \geqslant 3$.

Let $a_{i j}$ be Borel functions on $\mathbb{R}^{d}$ such that $a_{i j}(x)=a_{j i}(x)$ for all $i, j=1, \ldots, d$ and all $x \in \mathbb{R}^{d}$, and suppose there exists a constant $c_{21}>0$ such that

$$
c_{21}|y|^{2} \leqslant \sum_{i j}^{d} a_{i j}(x) y_{i} y_{j} \leqslant c_{21}^{-1}|y|^{2}
$$

for all $x, y \in \mathbb{R}^{d}$. The operator $L:=\frac{1}{2} \sum_{i, j}^{d} \partial_{i}\left(a_{i j} \partial_{j}\right)$ is called a uniformly elliptic operator in divergence form. Let $Y=\left(Y_{t}, \mathbb{P}^{x}\right)$ be the corresponding diffusion in the sense that for every $f \in C^{2}\left(\mathbb{R}^{d}\right)$ and every $x \in \mathbb{R}^{d}$, the process

$$
f\left(Y_{t}\right)-f\left(Y_{0}\right)-\int_{0}^{t} L f\left(Y_{s}\right) d s
$$

is a $\mathbb{P}^{x}$-martingale. 
Let $P_{t}^{Y} f(x):=\mathbb{E}_{i}^{x}\left[f\left(Y_{t}\right)\right]$ be transition operators of $Y$. It is well known that there exists a symmetric function $p^{Y}(t, x, y)$ such that $P_{t}^{Y} f(x)=\int p^{Y}(t, x, y)$ $f(y) d y$. The function $p^{Y}(t, x, y)$ is the heat kernel of the operator $L$ and it satisfies the following Gaussian bounds: There exists positive constants $c_{22}, c_{23}, c_{24}, c_{25}$ such that

$$
\left.\left.c_{22} t^{-d / 2} \exp \left\{-c_{23}|x-y|^{2} / t\right)\right\} \leqslant p^{Y}(t, x, y) \leqslant c_{24} t^{-d / 2} \exp \left\{-c_{25}|x-y|^{2} / t\right)\right\}
$$

for all $t>0$ and all $x, y \in \mathbb{R}^{d}$ (e.g., [11]). Let $G^{Y} f(x):=\mathbb{E}^{x} \int_{0}^{\infty} f\left(Y_{s}\right) \mathrm{d} s$ be the potential operator of $Y$. Then

$$
G^{Y} f(x)=\int_{0}^{\infty} \int_{\mathbb{R}^{d}} p^{Y}(t, x, y) \mathrm{d} y \mathrm{~d} t=\int_{\mathbb{R}^{d}} G^{Y}(x, y) f(y) \mathrm{d} y,
$$

where $G^{Y}(x, y):=\int_{0}^{\infty} p^{Y}(t, x, y) \mathrm{d} t$ is the Green function of $Y$. The function $G^{Y}$ is symmetric, and it follows immediately from (5.1) that there exists positive constants $c_{26}, c_{27}$ such that

$$
c_{26}|x-y|^{2-d} \leqslant G^{Y}(x, y) \leqslant c_{27}|x-y|^{2-d}
$$

for all $x, y \in \mathbb{R}^{d}$.

Let $T=\left(T_{t}: t \geqslant 0\right)$ be a subordinator independent of the diffusion $Y$ with Laplace exponent $\phi$, let $X_{t}:=Y\left(T_{t}\right), t \geqslant 0$, be the subordinate diffusion, and let $G(x, y)$ denote the Green function of $X$. By use of (5.1) we can easily obtain the following analogs of Theorem 3.1, respectively Theorem 3.3. If $\phi(\lambda) \sim b \lambda$, $\lambda \rightarrow \infty$, for $b>0$, or if $\phi(\lambda) \sim \gamma^{-1} \lambda^{\alpha / 2}, \lambda \rightarrow \infty, \alpha \in(0,2)$, and $\phi$ is a complete Bernstein function, then

$$
G(x, y) \asymp|x-y|^{\alpha-d}
$$

when $|x-y| \rightarrow 0$. If $\phi(\lambda) \sim \gamma^{-1} \lambda^{\alpha / 2}, \lambda \rightarrow 0, \alpha \in(0,2]$, and $\phi$ is a complete Bernstein function, then

$$
G(x, y) \asymp|x-y|^{\alpha-d}
$$

when $|x-y| \rightarrow \infty$. The asymptotic behavior of the Green function at zero implies that Propositions 3.5 and 3.7 and Lemma 4.1 are valid. We note that in the case of $\alpha / 2$-stable subordinator, Selmi [21] has established that the transition density of $Y$ subordinate with an $\alpha / 2$-stable subordinator is comparable to the transition density of symmetric stable processes. This implies that $G(x, y) \asymp$ $|x-y|^{\alpha-d}$ as $|x-y| \rightarrow 0$ and $|x-y| \rightarrow \infty$. 
In order to obtain the analogs of (4.5) and (4.6), besides (4.4), we have to assume that there is a positive constant $c_{28}$ such that

$$
\nu(t) \leqslant c_{28} \nu(2 t), \quad 0<t<\infty .
$$

(The assumption above is satisfied, for example, when $T_{t}=b t+\tilde{T}_{t}$ with $\tilde{T}$ being a stable subordinator.) Moreover, instead of one function $j$, we have to work with two such functions, because of different exponents in (5.1). Consequently, the proof of Lemma 4.4 requires minor changes. Proof of the Harnack inequality remains the same.

We further indicate extensions to some fractional diffusions. We refer the reader to [1] for background information on fractional diffusions. Let $F \subset \mathbb{R}^{d}$, $d \geqslant 3$, be a nonempty closed set such that $\operatorname{diam}(F)=\infty$, and let $d_{f} \in(0, d]$. A Borel measure $\mu$ is a $d_{f}$-measure on $F$ if there are constants $c_{29}, c_{30}>0$ such that

$$
c_{29} r^{d_{f}} \leqslant \mu(B(x, r)) \leqslant c_{30} r^{d_{f}}, \quad x \in F, r>0 .
$$

The set $F$ is called a $d_{f}$ set if $F=\operatorname{supp}(\mu)$ for some $d_{f}$ measure $\mu$. A fractional diffusion on $F$ is a Feller process $Y=\left(Y_{t}, \mathbb{P}_{x}\right)$ with continuous paths and the state space $F$ such that $Y$ has a symmetric transition density $p(t, x, y)=p(t, y, x)$, $t>0, x, y \in F$, which is jointly continuous for each $t>0$ and satisfies

$$
\begin{gathered}
c_{31} t^{-d_{s} / 2} \exp \left(-c_{32}\left(\frac{|x-y|}{t^{1 / d_{w}}}\right)^{d_{w} /\left(d_{w}-1\right)}\right) \leqslant p(t, x, y) \\
\leqslant c_{33} t^{-d_{s} / 2} \exp \left(-c_{34}\left(\frac{|x-y|}{t^{1 / d_{w}}}\right)^{d_{w} /\left(d_{w}-1\right)}\right)
\end{gathered}
$$

for all $t>0$ and all $x, y \in F$. Here $c_{31}, c_{32}, c_{33}, c_{34}$ are positive constants, $d_{w}>0$ and $d_{s}=2 d_{f} / d_{w}$. We assume that $d_{s}>2$ which implies that the process $Y$ is transient ([1], p. 40).

Let $T=\left(T_{t}: t \geqslant 0\right)$ be a subordinator, independent of $Y$, with a complete Bernstein Laplace exponent $\phi$. Let $u$ denote the potential density of $T$. Let $X_{t}:=Y\left(T_{t}\right)$ be the subordinate fractional diffusion. The case when $T$ is an $\alpha / 2$ stable subordinator was studied in $[16,26]$. The Green function of $X$ can be written as

$$
G(x, y)=\int_{0}^{\infty} p(t, x, y) u(t) \mathrm{d} t .
$$

By using the lower bound (5.3) for $p(t, x, y)$, and by change of variables, we can obtain the following lower bound for $G(x, y)$ :

$$
G(x, y) \geqslant c_{35}|x-y|^{d_{w}-d_{f}} \int_{0}^{\infty} s^{-d_{s} / 2+d_{f}-d_{w}} \mathrm{e}^{-s} u\left(\frac{c_{32}^{d_{w}-1}|x-y|^{d_{w}}}{s^{d_{w}}-1}\right) \mathrm{d} s,
$$

where $c_{35}>0$, and similarly for the upper bound. 
Assume that $\phi$ satisfies (3.3), i.e., $\phi(\lambda) \sim \gamma^{-1} \lambda^{\alpha / 2}$ as $\lambda \rightarrow \infty, \alpha \in(0,2]$. Similarly as in the proof of Theorem 3.1 we obtain that $G(x, y)$ satisfies

$$
G(x, y) \asymp|x-y|^{\frac{\alpha}{2} d_{w}-d_{f}}, \quad|x-y| \rightarrow 0 .
$$

If $\phi(\lambda) \sim \gamma^{-1} \lambda^{\alpha / 2}, \lambda \rightarrow 0, \alpha \in(0,2]$, and $\phi$ is a complete Bernstein function, the analog estimate is valid for $G$ as $|x-y| \rightarrow \infty$.

\section{Acknowledgements}

We thank Z.-Q. Chen for helpful discussions on the first version of this paper. We also thank the referee for the helpful comments. The research of Renming Song is supported in part by a joint US-Croatia grant INT 0302167. The research of Zoran Vondraček is supported in part by MZOS grant 0037107 of the Republic of Croatia and in part by a joint US-Croatia grant INT 0302167.

\section{References}

1. Barlow, M.T.: Diffusions on fractals, in Lecture Notes on probability theory and Statistics, École d' Été de Probabilités de Saint-Flour XXV - 1995, Lect. Notes Math. 1690, Springer, 1998, pp. 1-121.

2. Bass, R.F. and Kassmann, M.: 'Harnack inequalities for non-local operators of variable order', Trans. Amer. Math. Soc. 357 (2005), 837-850.

3. Bass, R.F. and Levin, D.A.: 'Harnack inequalities for jump processes', Potential Anal. 17 (2002), 375-388.

4. Bass, R.F. and Levin, D.A.: 'Transition probabilities for symmetric jump processes', Trans. Amer. Math. Soc. 354 (2002), 2933-2953.

5. Berg C. and Forst, G.: Potential Theory on Locally Compact Abelian Groups, Springer, Berlin, 1975.

6. Bertoin, J.: Lévy Processes, Cambridge University Press, Cambridge, 1996.

7. Bingham, N.H., Goldie, C.M. and Teugels, J.L.: Regular Variation, Cambridge University Press, Cambridge, 1987.

8. Bogdan, K., Stós, A. and Sztonyk, P.: 'Potential theory for Lévy stable processes', Bull. Pol. Acad. Sci., Math. 50 (2002), 361-372.

9. Chen, Z.-Q. and Kumagai, T.: 'Heat kernel estimates for stable-like processes on $d$-sets', Stoch. Process. Appl. 108 (2003), 27-62.

10. Chen, Z.-Q. and Song, R.: 'Drift transform and Green function estimates for discontinuous processes', J. Funct. Anal. 201 (2003), 262-281.

11. Davies, E.B.: Heat Kernels and Spectral Theory, Cambridge University Press, Cambridge, 1989.

12. Geman, H., Madan, D.B. and Yor, M.: 'Time changes for Lévy processes', Math. Finance 11 (2001), 79-96.

13. Jacob, N.: Pseudo Differential Operators and Markov Processes, Vol. 1, Imperial College Press, London, 2001.

14. Jacob, N. and Schilling, R.L.: 'Some Dirichlet spaces obtained by subordinate reflected diffusions', Rev. Mat. Iberoamericana 15 (1999), 59-91. 
15. Krylov, N.V. and Safonov, M.V.: 'An estimate of the probability that a diffusion process hits a set of positive measure', Sov. Math. Dokl. 20 (1979), 253-255.

16. Kumagai, T.: Some remarks for stable-like jump processes on fractals, in P. Grabner and W. Woess (eds), Trends in Math., Fractals in Graz 2001, Birkhäuser, Basel, 2002, pp. 185-196.

17. Riesz, M.: 'Integrals de Riemann-Liouville et potentiels', Acta Szeged 9 (1938), 1-42.

18. Ryznar, M.: 'Estimates of Green functions for relativistic $\alpha$-stable process', Potential Anal. 17 (2002), 1-23.

19. Sato, K.-I.: Lévy Processes and Infinitely Divisible Distributions, Cambridge University Press, Cambridge, 1999.

20. Schilling, R.L.: Zum Pfadenverhalten vom Markovschen Prozessen, die mit Lévy-Prozessen vergleichbar sind, Dissertation Universität Erlangen-Nürnberg, Erlangen, 1994.

21. Selmi, M.: 'Comparison des semi-groupes et des résolvantes d'ordre $\alpha$ associés à des opérateurs différentiels de type divergence', Potential Anal. 3 (1994), 15-45.

22. Song, R.: 'Sharp bounds on the density, Green function and jumping function of subordinate killed BM', Probab. Theory Related Fields 128 (2004), 606-628.

23. Song, R. and Vondraček, Z.: 'Potential theory of subordinate killed Brownian motion in a domain', Probab. Theory Related Fields 125 (2003), 578-592.

24. Song, R. and Vondraček, Z.: 'Harnack inequalities for some classes of Markov processes', Math. Z. 246 (2004), 177-202.

25. Song, R. and Vondraček, Z.: 'Harnack inequality for some discontinuous Markov processes with a diffusion part, Glas. Mat. 40 (2005), 177-187.

26. Stós, A.: 'Symmetric $\alpha$-stable processes on $d$-sets', Bull. Pol. Acad. Sci. Math. 48 (2000), 237-245.

27. Vondraček, Z.: 'Basic potential theory of certain nonsymmetric strictly $\alpha$-stable processes', Glas. Mat. 37 (2002), 193-215. 